\title{
Mucormicosis diseminada en un paciente diabético: un reporte de caso
}

\section{Disseminated mucormycosis in a diabetic patient: a case report}

Correspondencia

Natalia Gladys Vargas Herrera nataliavh84@hotmail.com

Recibido: 20/06/2019

Arbitrado por pares

Aprobado: 07/08/2019

Citar como: Vargas Herrera N, Saavedra Velazco $M$, Contreras Calero E, Ramos Castillo W, Mendo Urbina F, Pichardo Rodríguez R. Mucormicosis diseminada en un paciente diabético: un reporte de caso. Acta Med Peru. 2019;36(4):287-90.
Natalia Vargas Herrera ${ }^{1, a}$, Marcos Saavedra Velasco ${ }^{1, a}$, Elías Contreras Calero ${ }^{1, b}$, Wilder Ramos Castillo ${ }^{1, c}$, Fernando Mendo Urbina ${ }^{1, \mathrm{~d}}$, Rafael Pichardo Rodriguez ${ }^{1, \mathrm{e}}$

1 Hospital Nacional Edgardo Rebagliati Martins (HNERM). Seguro Social de Salud (EsSalud). Lima - Perú.

a Médico residente de Enfermedades Infecciosas y Tropicales; b Médico infectólogo; c Médico anátomo-patólogo; d Médico internista; e Médico-cirujano.

\section{RESUMEN}

La mucormicosis es una infección fúngica altamente mortal que se presenta en pacientes con algún grado de inmunosupresión, siendo la forma rinocerebral la más común. Es el primer reporte en el Perú de mucormicosis diseminada con compromiso multisistémico en un paciente con cetoacidosis diabética. Se presenta el caso de un varón de 47 años diabético procedente de la selva peruana con cuadro de insuficiencia respiratoria en ventilación mecánica. A su ingreso presenta leucocitosis, acidosis metabólica anion gap elevado, hiperglicemia e hipoalbuminemia. Posteriormente, es intervenido quirúrgicamente en tres oportunidades por presentar abdomen agudo con hallazgos en la patología de necrosis y perforación de varios órganos, falleciendo a los pocos días. Paciente se encontraba recibiendo su quinta dosis de anfotericina B deoxicolato. Se observaron hifas con angulación recta compatible con mucormicosis en estómago, intestino y pulmón.

Palabras clave: Mucormicosis; Mucorales; Zigomicosis; Cetoacidosis diabética (fuente: DeCS).

\section{ABSTRACT}

Mucormycosis is a highly lethal fungal infection occurring in patients with some degree of immunosuppression. The rhinocerebral form is the most frequent presentation. This is the first report in Peru of a case of disseminated mucormycosis in a patient with diabetic ketoacidosis. This is a 47-year-old diabetic male subject who was referred from the Amazon jungle and presented with respiratory insufficiency receiving mechanical ventilation. On admission, the patient had leukocytosis, metabolic acidosis with high anion gap, hyperglycemia, and hypoalbuminemia. Soon afterwards, the patient underwent surgery because of acute abdomen, and the anatomopathological examination revealed necrosis and hollow viscus perforation, and he ultimately died. At this time, he was receiving amphotericin B deoxycholate. Straight angle hyphae compatible with mucormycosis were found in stomach, intestine, and lungs.

Keywords: Mucormycosis; Mucorales; Zygomycosis; Diabetic ketoacidosis (source: MeSH). 


\section{INTRODUCCIÓN}

La mucormicosis es una infección angioinvasiva emergente y devastadora causada por los hongos filamentosos ubicuos de la orden Mucorales y clase Zigomicetos. Es la tercera micosis invasiva en orden de importancia luego de candidiasis y aspergilosis en pacientes severamente inmunocomprometidos (con neoplasias hematológicas, trasplantados de órganos sólidos o hematopoyéticos) ${ }^{[1,2]}$. También se presenta en pacientes con diabetes mellitus mal controlada, neutropenia, traumatismos, uso de deferoxamina y corticoterapia ${ }^{[3]}$.

La histopatología distintiva de la mucormicosis es la necrosis tisular resultante de la angioinvasión y posterior trombosis ${ }^{[1]}$. Se clasifica según su presentación clínica en: rinocerebral, pulmonar, cutánea, gastrointestinal, diseminada y miscelánea ${ }^{[4]}$, siendo la rinocerebral la forma más frecuente de mucormicosis en pacientes con diabetes mellitus ( $66 \%$ de casos), mientras que la diseminada se describe en quemados, neutropénicos o en tratamiento con deferoxamina ${ }^{[5,6]}$.

Presentamos el caso inusual de un varón diabético que desarrolló mucormicosis diseminada con afectación pulmonar, gástrica, intestinal, diafragmática, hepática, renal, suprarrenal, pancreática, pericárdica, aortica y cutánea.

\section{REPORTE DE CASO}

Varón de 47 años, natural y procedente de la selva peruana, con antecedente de diabetes mellitus desde los 16 años de edad, mal controlado y polineuropatía diabética de dos meses de evolución a pesar de tratamiento con metformina y glibenclamida. Inicia con rinorrea, tos y cefalea por dos semanas, posteriormente fiebre y convulsión tónico clónica generalizada. Ingresa a un servicio de emergencia de un hospital regional, con Glasgow 10/15, donde la tomografía axial computarizada (TAC) cerebral y estudio de líquido cefalorraquídeo son normales.

Por evolución tórpida y mal patrón respiratorio entra en ventilación mecánica y es referido al Hospital Nacional Edgardo Rebagliati Martins, en Lima, con diagnóstico de cuadriplejía arrefléxica más neumonía bilateral. A su ingreso resaltaban crépitos en hemitórax izquierdo, pero se encontraba en normopeso, despierto y obedeciendo órdenes simples. Funciones vitales al ingreso: PA 150/80 mmHg, FC 90 x min, FR 34 x min, hemoglobina $10,3 \mathrm{~g}$, leucocitos $20,4 \times 10^{3} / \mathrm{uL}$, albúmina $1,9 \mathrm{~g} /$ dL, glucosa $197 \mathrm{mg} / \mathrm{dL}$ y HbA1\%: 9,4\%. La TAC torácica informó consolidado bilateral con nódulo pulmonar izquierdo, nueva TAC cerebral sin alteraciones. Recibe cobertura antibiótica empírica con vancomicina y meropenem.

Una semana después cursa con abdomen agudo quirúrgico y melena requiriendo laparotomía exploratoria, encontrándose necrosis más perforación de fondo y cuerpo gástrico, por lo que se realiza gastrectomía total. Se inicia anfotericina $B$ deoxicolato $1 \mathrm{mg} / \mathrm{Kg}$ cada 24 horas debido a la mala respuesta a la antibioticoterapia. Una semana después de la primera cirugía se le realiza resección de colon transverso y sigmoides por perforación de ángulo esplénico. Al quinto día es reintervenido quirúrgicamente con hallazgos: necrosis de pared abdominal, duodeno, bazo y páncreas, falleciendo a los pocos días por falla multiorgánica.

El estudio de anatomía patológica de las piezas operatorias del estómago y colon (Figura 1) indica necrosis isquémica con severo infiltrado inflamatorio y trombos vasculares en cuya luz y pared se aprecian múltiples estructuras micóticas, anchas, sin septos, con hifas poco ramificadas y angulación en $90^{\circ}$ y cuyas características morfológicas corresponden a mucor sp (Figuras 2 y 3 ). La necropsia informa proceso infeccioso micótico necrotizante multisistémico, morfológicamente compatible con mucormicosis invasiva diseminada. Hubo compromiso de pulmón, intestino, estómago, diafragma, hígado, riñón, glándula suprarrenal, pericardio, aorta, páncreas y bordes de la herida operatoria. Adicionalmente, se encontró aspergilosis pulmonar, con cultivos bacterianos negativos.

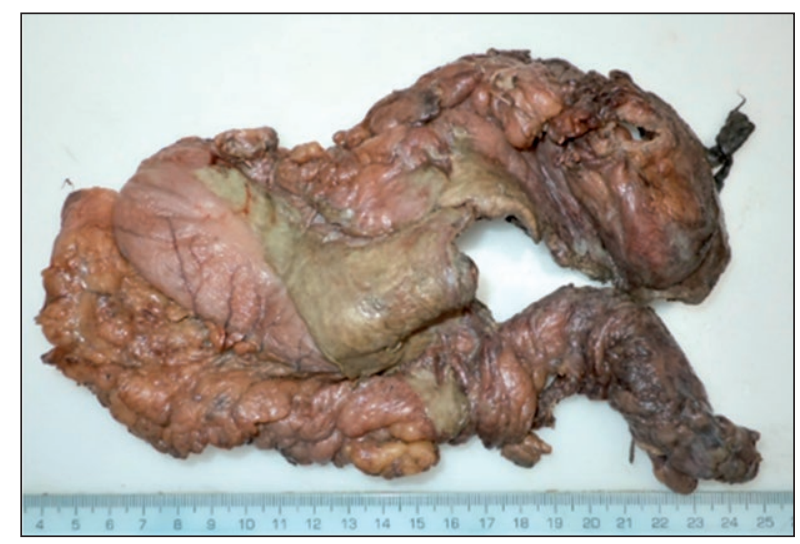

Figura 1. Macroscopía de pared gástrica con extensa perforación, bordes necróticos y áreas cubiertas por fibrina.

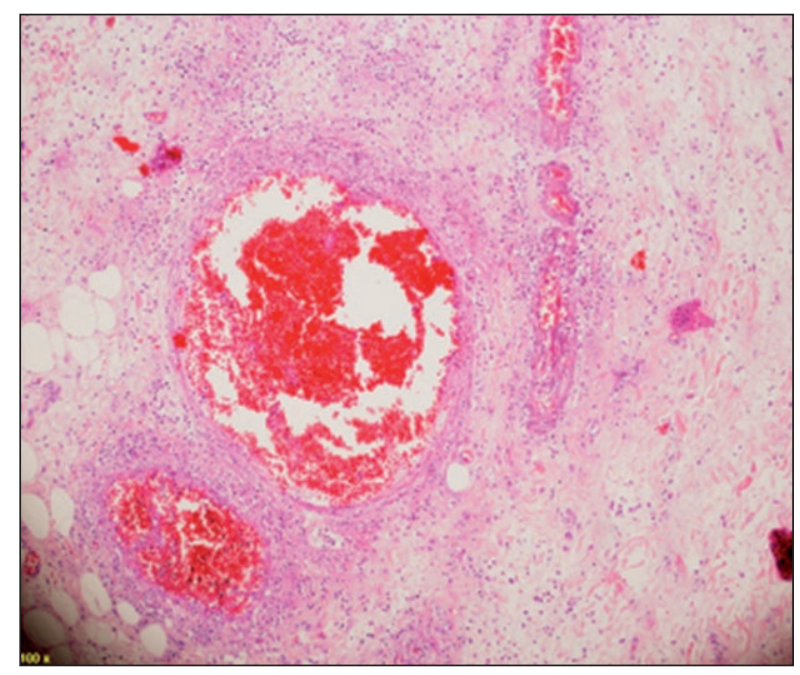

Figura 2. Microscopía de pared gástrica con presencia de trombos vasculares en cuya luz y pared se aprecian estructuras micóticas compatibles con Mucor spp. 


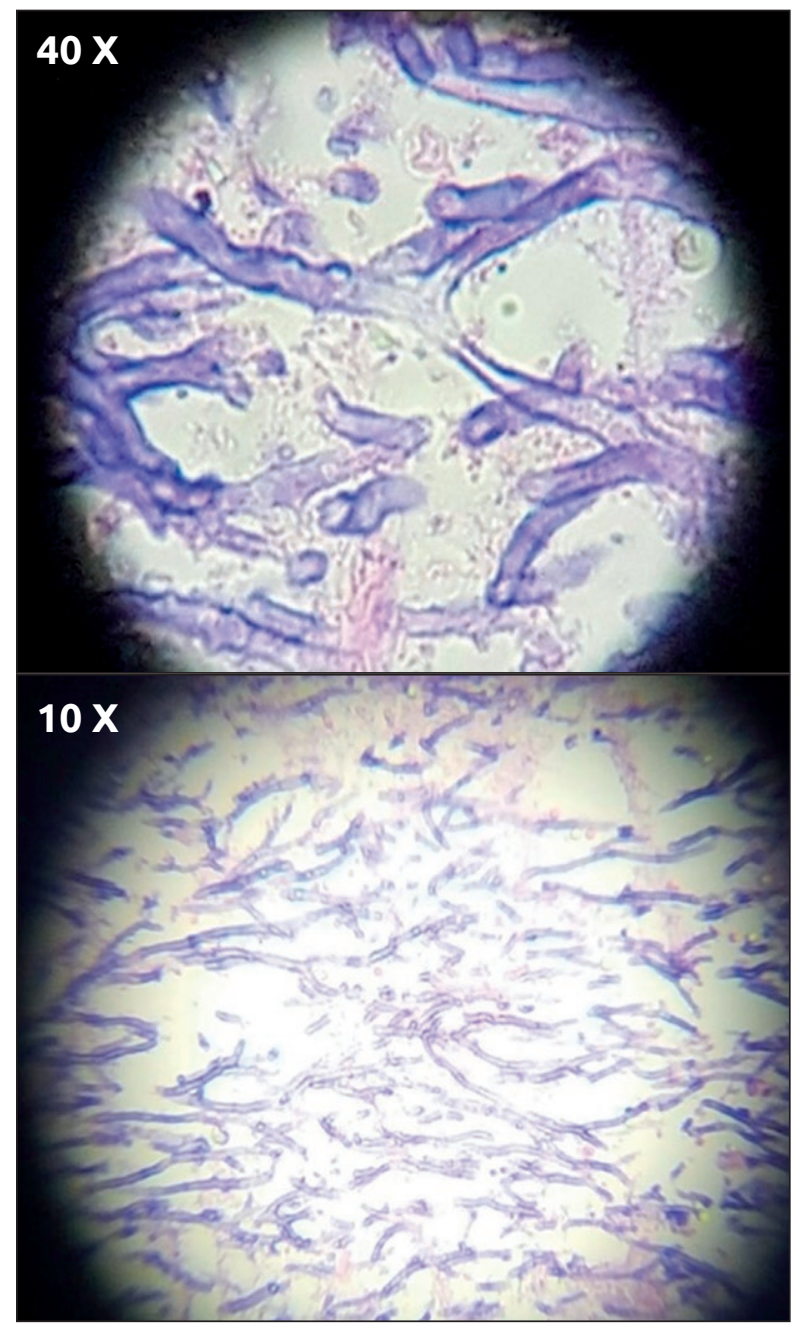

Figura 3. Microscopía de parénquima pulmonar con presencia de hifas en ángulo recto, poco ramificadas, anchas, sin septos, algunas con angulación en $90^{\circ}$ cuyas características morfológicas son compatibles con Mucor spp.

\section{DISCUSIÓN}

La mucormicosis diseminada es una entidad rara, sin embargo a pesar de su difícil diagnóstico la incidencia está en aumento ${ }^{[7]}$. Su tasa de mortalidad se acerca al $100 \%$ y ocurre principalmente en pacientes con neoplasias hematológicas o post-trasplantados, quemados, prematuros y en tratamiento con deferoxamina, en países desarrollados ${ }^{[8]}$. Su presentación clínica es inespecífica e involucra a menudo dos o más sistemas no contiguos como el cerebro y pulmón y usualmente se diagnostica post-mortem debido a su alta mortalidad ${ }^{[9,10]}$.

En una serie de 929 casos de mucormicosis, Roden et al. mostraron que la enfermedad diseminada se presentó en 40 $50 \%$ de pacientes con mucormicosis cerebral o pulmonar, siendo la tasa de mortalidad $96 \%{ }^{[11]}$.
La asociación entre mucormicosis y diabetes no es del todo comprendida pero es probable que se deba a los efectos multifactoriales de la hiperglicemia y la cetoacidosis que deprimen el sistema inmune fagocitario proporcionando un entorno facultativo para la proliferación fúngica ${ }^{[5,6]}$. Además, se incrementa el hierro sérico libre, esencial para el metabolismo fúngico, mientras que un $\mathrm{pH}$ ácido disminuye la respuesta inmune innata frente al hongo. Asimismo, los receptores del huésped encargados de invadir y dañar a las células epiteliales humanas por parte del Rhizopus aumentan su expresión ${ }^{[12,13]}$. Finalmente, la cetoacidosis disminuye la permeabilidad de la barrera hematoencefálica contribuyendo a la predilección por la enfermedad rinocerebral en esta población ${ }^{[14]}$. En un estudio descriptivo de 221 casos de mucormicosis en México, se encontró que en pacientes diabéticos con cetoacidosis la mortalidad fue mayor que en diabéticos sin cetoacidosis ${ }^{[15]}$.

El tratamiento consta de tres puntos importantes: terapia antifúngica temprana, cirugía y manejo de las comorbilidades ${ }^{[16]}$ La anfotericina B (formulación lipídica) es la terapia de elección, siendo la dosis sugerida $5-10 \mathrm{mg} / \mathrm{kg} /$ día en infección del sistema nervioso central. Posaconazol e isavuconazol también son agentes activos de primera línea, mientras que combinaciones de anfotericina $B$ liposomal con caspofungina o posaconazol son opciones para el tratamiento de rescate ${ }^{[17]}$. Asimismo, Roden et al. demostraron en su análisis multivariado que la asociación de terapia antifúngica con cirugía aumentó significativamente las tasas de supervivencia (69\%), mientras que el $97 \%$ de pacientes sin tratamiento fallecieron ${ }^{[11]}$, esto es respaldado por reportes como el de Sipsas et al. ${ }^{[18]}$.

No obstante, la mucormicosis diseminada es una infección fúngica letal a pesar de la instauración terapéutica temprana, siendo infrecuente llegar al diagnóstico antes del fallecimiento debido a su alta agresividad, rápido avance y la baja sensibilidad de los hemocultivos ${ }^{[19,20]}$.

La limitación más importante fue el no contar con anfotericina B liposomal debido al desabastecimiento de dicho medicamento en el hospital, sin embargo, se le administró la presentación deoxicolato. La fortaleza más importante fue contar con la confirmación del diagnóstico por anatomía patológica.

Por ende, se concluye que la mucormicosis diseminada puede presentarse en pacientes con diagnóstico de diabetes mellitus a pesar de que en ellos prevalece la forma rinocerebral. Por tal motivo, es esencial tener un alto índice de sospecha clínica en pacientes con factores de riesgo de infección fúngica que presenten deterioro clínico a pesar de la terapia antibacteriana de alto espectro, como en el caso del paciente presentado.

\section{Fuentes de financiamiento: Autofinanciado.}

Conflictos de Interés: Los autores declaran no tener conflictos de interés. 


\section{REFERENCIAS BIBLIOGRÁFICAS}

1. Petrikkos G, Skiada A, Lortholary O, Roilides E, Walsh TJ, Kontoyiannis DP. Epidemiology and clinical manifestations of mucormycosis. Clin Infect Dis. 2012;54 Suppl 1:S23-34.

2. Lanternier F, Dannaoui E, Morizot G, Elie C, Garcia-Hermoso D, Huerre $\mathrm{M}$, et al. A global analysis of mucormycosis in France: the RetroZygo Study (2005-2007). Clin Infect Dis. 2012;54 Suppl 1:S35-43.

3. Ibrahim AS, Spellberg B, Walsh TJ, Kontoyiannis DP. Pathogenesis of mucormycosis. Clin Infect Dis. 2012;54 Suppl 1:S16-22.

4. Prabhu RM, Patel R. Mucormycosis and entomophthoramycosis: a review of the clinical manifestations, diagnosis and treatment. Clin Microbiol Infect. 2004;10 Suppl 1:31-47.

5. Singh V, Singh M, Joshi C, Sangwan J. Rhinocerebral mucormycosis in a patient with type 1 diabetes presenting as toothache: a case report from Himalayan region of India. BMJ Case Rep. 2013;2013:bcr2013200811.

6. McCrory MC, Moore BA, Nakagawa TA, Givner LB, Jason DR, Palavecino EL, et al. Disseminated Mucormycosis in an Adolescent with Newly Diagnosed Diabetes Mellitus. Pediatr Infect Dis J. 2014;33(10):1094-6.

7. Van Lieshout M. Disseminated mucormycosis: (almost) fatal! Neth J Crit Care. 2017;25(3):104-7.

8. Kauffman CA, Malani AN. Zygomycosis: an emerging fungal infection with new options for management. Curr Infect Dis Rep. 2007;9(6):435-40.

9. Wang $Q$, Liu B, Yan Y. Disseminated mucormycosis (DM) after pneumonectomy: a case report. BMC Infect Dis. 2016;16(1):337.

10. Lee D-G, Choi J-H, Choi S-M, Yoo J-H, Kim Y-J, Min C-K, et al. Two cases of disseminated mucormycosis in patients following allogeneic bone marrow transplantation. J Korean Med Sci. 2002;17(3):403-6.
11. Roden MM, Zaoutis TE, Buchanan WL, Knudsen TA, Sarkisova TA, Schaufele RL, et al. Epidemiology and outcome of zygomycosis: a review of 929 reported cases. Clin Infect Dis. 2005;41(5):634-53.

12. Casqueiro J, Casqueiro J, Alves C. Infections in patients with diabetes mellitus: A review of pathogenesis. Indian J Endocrinol Metab. 2012;16 Suppl 1:S27-36.

13. Peleg AY, Weerarathna T, McCarthy JS, Davis TME. Common infections in diabetes: pathogenesis, management and relationship to glycaemic control. Diabetes Metab Res Rev. 2007;23(1):3-13.

14. Lanternier F, Lortholary 0 . Zygomycosis and diabetes mellitus. Clin Microbiol Infect. 2009;15 Suppl 5:21-5.

15. Corzo-León DE, Chora-Hernández LD, Rodríguez-Zulueta AP, Walsh TJ. Diabetes mellitus as the major risk factor for mucormycosis in Mexico: Epidemiology, diagnosis, and outcomes of reported cases. Med Mycol. 2018;56(1):29-43.

16. Chayakulkeeree M, Ghannoum MA, Perfect JR. Zygomycosis: the re-emerging fungal infection. Eur J Clin Microbiol Infect Dis. 2006;25(4):215-29.

17. Skiada A, Lass-Floerl C, Klimko N, Ibrahim A, Roilides E, Petrikkos G. Challenges in the diagnosis and treatment of mucormycosis. Med Mycol. 2018;56(suppl 1):93-101.

18. Sipsas NV, Gamaletsou MN, Anastasopoulou A, Kontoyiannis DP. Therapy of Mucormycosis. J Fungi. 2018;4(3):90.

19. Salgado-Camarillo J, Morales-Pogoda I, Rugerio-Trujillo A. Mucormycosis, case report and review of literature. Rev Sanid Mil. 2017;70(3):308-12.

20. Sánchez-Sánchez RA, Sánchez-Goytia V, García-Peña A, Cortés García BY, Pérez-Reyes JA, Escobar-Martínez N, et al. Mucormicosis diseminada. Presentación de caso y revisión de la literatura. An Radiol México. 2016;15(1):34-8.

\section{Ahora puede enviar sus artículos para Acta Médica Peruana en nuestro Open Journal System:}

\section{www.amp.cmp.org.pe}

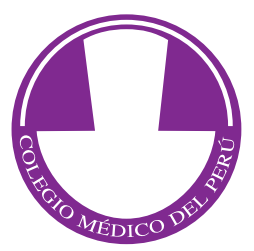

